\title{
Mass Campaign as an Instrument of Endemic Disease Control in Developing Countries*
}

\author{
CHIEF SIR SAMUEL MANUWA,† KT., C.M.G., O.B.E., M.D., F.R.C.P.ED., F.R.C.S.ED., F.R.S.ED.
}

Brit. med.7., 1968, 4, 634-636

\begin{abstract}
"In the underdeveloped areas... release of the countries from the tangled undergrowth of mass disease is a prerequisite of development. Fust as the Panama Canal could not be constructed till Gorgas had mastered yellow fever, so the technical resources of these countries cannot be effectively exploited until the conditions of better health have been created. . . . Somehow governments will have to finance the capital requirements of health. . . ."UNITED NATIONS (1952).
\end{abstract}

\section{Introduction}

Two main problems beset the health administrator in developing countries. The first is the chronic shortage of trained manpower and of financial resources. The second is that of the peculiar health pattern and of the epidemiology of disease in those countries. The two are intimately related.

The peculiar epidemiology in these regions is that presented by the various endemic communicable diseases; and the speed, extent, degree, and efficiency with which the "tangled undergrowth" which they each and severally constitute are controlled and eradicated largely determine conservation of manpower resources and the rate and progress of economic development and growth. Chief among these diseases are malaria, sleeping sickness, yaws, yellow fever, leprosy, tuberculosis, trachoma, venereal disease, onchocerciasis, measles, smallpox, cerebrospinal fever, ancylostomiasis, bilharziasis, poliomyelitis, and so on.

In developing countries communicable diseases are community-wide in prevalence and incidence. They occur in the mass, and must therefore be dealt with largely in the mass, by means of mass campaigns.

\section{Mass Campaigns}

A mass campaign is a special exercise in public health practice, and its purpose is to cure, control, and eradicate endemic communicable diseases by specific measures applied on a community-wide basis. These measures must embrace a total (that is, $100 \%$ ), systematic, and rapid geographical coverage of the population at risk, and also of future additions to that population by birth, immigration, or both.

Mass screening, in what has been termed Predictive Medicine, is not considered in this paper.

- An abridged version of a paper presented to the Eighth Internationa Congresses on Tropical Medicine and Malaria at Teheran, Iran, in Congresses on 1968.

+ Pro-Chancellor and Chairman of Council of the University of Ibadan Formerly Inspector-General of Medical Services and Chief Medica Adviser to the Government of the Federation of Nigeria. Present address: Federal Public Service Commission, Lagos, Nigeria.

\section{Principal Aim}

The principal aim of a mass campaign is to achieve the speedy interruption of the cycle of transmission of the disease under attack. This is done in various ways :

(1) By exterminating the disease vector or its larvae by chemical means, which include the use of residual insecticides and larvicides, and by biological methods such as parasitization, predators, and genetic control by selective mating.

(2) By destruction of the causative parasite in the human or intermediate host by specific chemotherapy, so as to reduce or liquidate the reservoir of active infection.

(3) By destruction of the intermediate host by chemical or other agents.

(4) By increasing resistance in the non-immune host by means of immunotherapy.

(5) By improving general measures of environmental sanitation, and by ecological and engineering measures designed to avoid conditions favourable to the propagation and continuance of transmission.

In addition to total population coverage, these measures to be maximally effective must be applied at scheduled regular intervals, must be simple and standardized, and must be continually supervised throughout the campaign's entire duration.

The campaign must be centrally controlled by a specialist epidemiologist. It must be carried out simultaneously in all areas of the same country ; and it must so far as is possible be co-ordinated and synchronized with similar campaigns being carried out in neighbouring countries. Success of a campaign in one country may be marred by introduction of infection from an adjacent untreated territory.

It must of course be assumed that the operating country had carried out a preliminary audit (if necessary with external assistance) and had "counted the cost" in money and manpower resources. This is to ensure that it can afford not only to carry out the campaign but also to make certain that the campaign remains uninterrupted from any avoidable cause until it is finally completed.

If resources permit, multiple campaigns against more than one communicable disease may be organized concurrently. The sequential method of attacking one disease at a time is, however, the more economical. It is also the more effective in ultimately providing an increasing reservoir of trained polyvalent staff and thus gradually enriching a growing health service which had poor initial resources. Some fine health services in developing countries have been built up in this way.

In more recent years the World Health Organization has carried out, in concert with national governments, successful eradication programmes, notably against yaws, and currently against malaria, measles, and smallpox on a global basis. The 
Organization has enunciated certain principles of methodology of eradication, which will now be described.

\section{Principles of Methodology of Eradication}

Eradication of an endemic communicable disease is regarded as having been achieved when three factors at the end of a campaign against it are satisfied-namely, (1) the reservoir of infectious cases has been eliminated; (2) its epidemiological cycle of transmission is permanently broken; and (3) there is no further recrudescence of indigenous cases.

To achieve eradication the campaign must pass successively and successfully through the following four phases. The details of these phases are, however, flexible and could be adapted to the needs, resources, and epidemiology in any particular country.

\section{First Phase (Pre-eradication)}

A basic preparatory Pre-eradication Phase, aimed at providing a sound infrastructure of general health services, especially peripheral health services, with which the campaign will be integrated in its last stages. Peripheral health services include dispensaries, health centres, and other similar units in both rural and urban areas.

The preliminary establishment of this basic infrastructure of an acceptable minimum of central, intermediate, and peripheral network of essential health services is vital to the ultimate success of the entire operation. Without it complete eradication cannot be achieved. The minimum elements of the basic infrastructure are the provision of adequate maternal and child welfare ; general communicable disease control and environmental sanitation; maintenance of records for statistical purposes ; health education of the public; public health (community) nursing; a laboratory service; and medical care.

Concurrent with this general preparation will be specific preparation for the disease under attack. This would include provision for the collection of logistical and epidemiological data provided by surveys of the operational area; the mobilization and training of the appropriate specialized staff against the particular disease; the assembly of adequate quantities of medical supplies (potent drugs, thermostable vaccines, etc.) and of equipment (mechanical, vehicular, and clinical), with facilities for their continuous replenishment and repair ; excellent public relations and propaganda; and the testing of the organization in experimental and demonstration projects, in trial against D Day.

\section{Second Phase (Attack)}

The essential features of this actual combat stage are maintenance of adequate and continuous supplies, of lines of communications, and of skilled supervision of subordinate and intermediate staff.

This stage may take one of two forms, depending on the nature of the programme and of the disease. In a short-term "crash" programme, as in yaws or malaria eradication by residual spraying, the object is to deliver within a scheduled time-table a "knock-down" blow in a systematic and rapid blitz, with $100 \%$ community coverage, and on an individual house-to-house basis. On the other hand, in long-term campaigns, as in tuberculosis and leprosy, the epidemiology of which is different, the attack takes the form of a siege of attrition, with the operation continued over a prolonged period, with contact maintained with the community over a number of years, with thorough case- and contact-finding, and sustained ambulant or domiciliary specific therapy.

A note of caution is necessary here. Where a combined multivalent vaccine or two separate vaccines are to be admini- stered simultaneously in a mass campaign (as in combined smallpox/measles and smallpox/yellow fever vaccination for example), care must be taken that neither of the two vaccines produces too severe a reaction; otherwise this may affect the success of the entire campaign. To ensure the greatest possible effectiveness and popular appeal the drugs and vaccines used must be minimally toxic and maximally potent.

\section{Third Phase (Consolidation and Surveillance)}

This phase is designed to eliminate any residual foci of infection. It succeeds the completed attack phase and ends when the disease has been completely eradicated. Though the staff of the general health services should have been active participators and collaborators with the special staff of the campaign from its very beginning, it is at this stage that the actual integration of the two branches should begin.

\section{Fourth Phase (Maintenance)}

This begins after eradication, and its object is continuous watchfulness in order to prevent recrudescence. Perhaps in no other single sphere of health activity is the saying that "eternal vigilance is the price of freedom " truer than in this phase of endemic disease control and eradication. For example, in West Africa in recent years relaxation of proper surveillance and maintenance has resulted in focal resurgence of yaws and yellow fever in areas where they had been eradicated.

Because this phase requires the coverage of large geographical areas in scouting for and the discovery of only one or two isolated residual or imported cases among a large population, the use as an "army of occupation" of the original specialized team which conducted the attack phase would manifestly be unjustified as being wasteful and expensive. It is also at this stage that the community and the local authorities may begin to lose their original interest and enthusiasm, or demand that attention be diverted to other health activities. The responsibility for executing this phase therefore rests fairly and squarely on the shoulders of the staff of the intermediate and peripheral rural and urban units, with their polyvalent functions. Final integration of this staff with the specialized staff of mass campaigns should not be difficult, as the latter would have themselves acquired polyvalent competence by the time they shall have taken part in various sequential campaigns. It is desirable, however, that a small nucleus of the original specialist staff be preserved and their training maintained.

\section{Other Side of the Coin}

Let us examine the other side of this unique and valuable coin of eradication of endemic disease by mass therapy.

Fears have been expressed that eradication programmes by mass campaigns are a double-edged sword which creates something of a dilemma for planners of national health services in developing countries. On the one hand, this weapon decreases the morbidity and mortality rates. But, on the other hand, it also increases the birth rate and life expectancy.

Various developing countries, notably India, have already taken energetic steps to counter this problem by health education and by establishing family planning as an important item in national governmental health policy.

In Africa, however, many parts, for their size, are still underpopulated. The problem in those particular parts is therefore one of ensuring increased manpower, particularly skilled manpower, in order to create the technological and economic capacity which will enable them to catch up with the more advanced countries of the world. So far, it is for them a question of a balanced rather than that of an unwanted population; but even in those parts the problem of overpopulation will at 
some future date inevitably arise, and we must begin to plan for it now. Nevertheless, its future emergence cannot be allowed to hold up the control and eradication of endemic disease, because the promotion of health and prevention of disease is a basic human right, and it is recognized that control and eradication programmes must proceed concurrently with equally vigorous programmes of improved agricultural productivity and health education.

\section{Conclusion}

The mass campaign is among the best tools in our armamentarium for the control and eradication of communicable mass disease. Its use has brought untold benefit to millions in the underdeveloped and underprivileged regions of the world by preventing disease, promoting and maintaining health, and improving living standards. Internationally, it has considerably reduced the risk of transmission of exotic and dangerous disease to other parts of the world.

Scourges and pestilences of diseases which had decimated whole populations in developing countries in the past are now almost unknown in those forms. Twenty years ago yellow fever was one of the major endemic and epidemic diseases in tropical Africa. Now it has almost disappeared. Smallpox and sleeping sickness have been or are being brought under control, and encouraging progress is being made against measles, poliomyelitis, tuberculosis, leprosy, and other diseases.

Unfortunately, however, many diseases remain a threat to millions. Cholera is still a menace. Though malaria has now been eradicated from a few countries, the fight against it has not progressed in other countries, notably in tropical Africa, as decisively as might be desired. The fight continues, however, and will eventually be successfully concluded.

Not the least of the contributions which mass campaigns have made to world health is that of improving the efficiency and standards of overall national health programmes. Successful campaigns against the most easily eradicated endemic diseases, such as yaws, are perhaps the most spectacular and effective means of arousing health consciousness and maintaining public confidence in the health services, particularly in backward communities. They are a useful means also of creating a reservoir of trained auxiliary staff.

Expenditure on mass campaigns is a sound investment in health. It yields rich dividends. The health services, particularly in developing countries, are the foundation stone of all social, educational, and, above all, economic development. A country's economy can be built only on the shoulders of the quick, the hale, and the hearty. It can never be built on the shoulders of the sick, the lame, and the dead.

\title{
HOSPITAL TOPICS
}

\section{New Self-administered Medical Questionary}

\author{
J. ANDERSON,* M.D., F.R.C.P. ; J. L. DAY, $†$ M.B., M.R.C.P.
}

Brit. med. F., 1968, 4, 636-638

A new comprehensive medical questionary has been devised in an attempt to assess the merits of a questionary in saving time and eliciting forgotten symptoms, with a view ultimately to establishing priority of medical need, identifying relevant symptoms, planning consultation, and, in the research field, elucidation of the incidence of symptoms, epidemiology, and genetic factors.

To date the medical questionaries that have been most widely used are the Cornell Medical Index and its successor, the Medical Data Index (Brodman et al., 1951 ; Brodman and van Woerkom, 1966), and in the psychiatric field the Maudsley Personality Index and the Eysenck Personality Index, all of which have been well validated (Eysenck and Eysenck, 1964) but limited in scope. Paul Hall (personal communication, 1968), in Scandinavia, has extended the experience of questionaries to outpatient investigation. A number of theoretical disadvantages of printed form questionaries are well known, and include failure of patients to complete questionaries of undue length, difficulty in analysing the mass of data, interference in doctor-patient relationship, and difficulty, with a fixed layout, in orientating patients towards appropriate symptoms.

A number of systems have been devised to assist a patient through a printed form questionary. Most recently, Mayne et al. (1968) described an experimental graphic display terminal in which photographic images are projected on a screen, the

\footnotetext{
* Professor of Medicine and Honorary Physician, King's College Hospital Medical School, London S.E.5.

† Medical Registrar, King's College Hospital, London S.E.5.
}

patient records a response with a light pen, and by means of I.B.M. 7040 and 1050 computers the data are analysed so that a maximum of 302 questions could be asked with good patient performance and a satisfactory narrative type history obtained.

In the design of this questionary these problems were taken into consideration. In this pilot study we attempted to find out more about these problems, and if they could be overcomé.

\section{Material and Methods}

The medical questionary was designed to include as many questions as were thought necessary to cover the important facts within a medical history, including all the major systems, general health, previous illness, operations, drugs, and family and social history. Throughout, the layout was such that ultimately computer analysis would be possible and alterations to individual questions feasible. A total number of 531 questions were created, grouped into sections (Table I). In order to assist the patients through such a large number of questions, a branching

TABLE I.-Make-up of Questionary

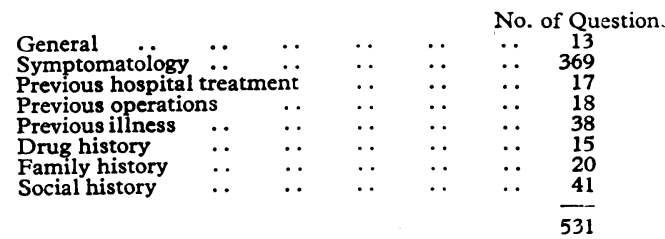

